\section{Un desafío a la justicia social peronista: la hidatidosis en la provincia de Buenos Aires, 1946-1952}

\author{
A challenge to
} Peronism's social justice: hydatidosis in the province of Buenos Aires, 1946-1952

\section{Adriana Valobra}

Departamento de Historia de la Universidad Nacional de La Plata, 48 s/n, entre 6 y 7 ,

1900 La Plata - Buenos Aires - Argentina indivalobra@hotmail.com
VALOBRA Adriana. Un desafío a la justicia social peronista: la hidatidosis en la provincia de Buenos Aires, 1946-1952. História, Ciências, Saúde - Manguinhos, Rio de Janeiro, v.14, n.4, p.1357-1375, out.-dez. 2007.

Analiza las políticas sanitarias del gobierno de Domingo Alfredo Mercante (1946-1952) mano derecha de Juan Domingo Perón - en la provincia de Buenos Aires, Argentina, atendiendo a lo que fue una de sus más instigantes líneas de intervención: la erradicación de la hidatidosis. Visibilizando un problema largamente olvidado por la historiografía, especificaremos de qué modo el gobierno mercantiano colocó a la hidatidosis en la agenda estatal a través de una legitimación estadística, socioeconómica y simbólica. Luego, especificaremos las estrategias de esta gestión: sanción de leyes regulatorias contra la endemia, creación de instituciones específicas antihidatídicas estatales, generación de espacios de información y educación interdisciplinaria, interministerial y hasta supranacionales con el fin de alcanzar el mayor conocimiento sobre la enfermedad e intercambiar experiencias que enriquecieran su propia práctica y, finalmente, la definición de acciones enfocadas en el relevo, tratamiento y profilaxis de animales y personas.

PALABRAS-CLAVE: políticas públicas; salud; hidatidosis; Mercante; peronismo.

VALOBRA, Adriana. A challenge to Peronism's social justice: hydatidosis in the province of Buenos Aires, 1946-1952. História, Ciências, Saúde - Manguinhos, Rio de Janeiro, v.14, n.4, p.1357-1375, Oct.-Dec. 2007.

This analysis of the sanitary policies enforced in the province of Buenos Aires by Domingo Alfredo Mercante-Juan Domingo Perón's right-hand man - focuses on one of his most fascinating efforts: the eradication of hydatidosis. In exploring an issue largely forgotten by historiography, the article describes how the Mercante administration (1946-52) used statistical, socioeconomic, and symbolic legitimization to place hydatidosis on the State agenda. The administration's strategies are also pinpointed: the passing of regulatory laws against this endemic disease; the creation of specific state anti-hydatidosis institutions; the creation of space for information and for interdisciplinary, interministerial, and even supranational education aimed at achieving greater knowledge of the disease and at the exchange of experiences; and, lastly, the definition of initiatives focused on relief, treatment, and prevention in animals and people.

KEYWORDS: public policy; health; hydatidosis; Mercante; Peronism. 
E lobjetivo de este trabajo es analizar las políticas sanitarias del gobierno de Domingo Alfredo Mercante - mano derecha del presidente argentino Juan Domingo Perón - en la provincia de Buenos Aires entre 1946 y 1952, atendiendo a lo que fue una de sus más instigantes intervenciones. Nos referimos a la erradicación de una zoonosis (enfermedades de los animales que se transmiten a los seres humanos) que afecta principalmente a las zonas rurales: la hidatidosis (Valobra, 2005). En efecto, la acción mercantiana antihidatídica fue una de las líneas de acción características de este gobierno que lo diferenciaron de las tareas realizadas en el ámbito nacional. En cierto sentido, fue una audaz apuesta que - con aciertos y limitaciones - logró cierta repercusión en el cono sur.

Entonces, especificaremos la singularidad de esta gestión y contribuiremos a visibilizar un problema largamente olvidado por la historiografía argentina que, a diferencia de la brasileña, está centrada en las epidemias urbanas y sólo recientemente ha dado frutos en la sanidad rural. Esto es llamativo pues en las fuentes se registran preocupaciones por el ámbito rural enlazado simbólicamente a la salud-enfermedad y al ideario nacionalista (Armus, 2005; Ballent, Gorelik, 2001).

Nuestra hipótesis es que el gobierno de Mercante, a través de la lucha antihidática del Ministerio de Salud y Acción Social, logró diferenciarse de sus predecesores en, al menos, cuatro acciones. Una, colocó en la agenda pública la hidatidosis como una enfermedad de cuidado y, al hacerlo, ofreció nuevas lógicas de racionalidad que legitimaron la importancia de esta dolencia al tiempo que impugnaron ciertas características socioeconómicas de la provincia. Dos, recuperó a la población rural como sujeto de las políticas estatales y, al mismo tiempo, construyó un nuevo estereotipo de poblador rural en el que se contrapuso por una parte, el del pasado, anclado en la miseria, ignorancia y enfermedad; y por otra, el del futuro, al que debía tenderse, pilar de la política de justicia social que trocaba su situación a la opulencia, conocimiento y salud. Tres, apeló a la interdisciplinariedad médico-veterinaria para prevenir y tratar la enfermedad y a la educación sanitaria para la propagación de nuevos modelos de higiene. Finalmente, propició la formación de redes internacionales en la consecución de sus objetivos.

\section{La hidatidosis en la agenda estatal bonaerense}

Desde principios de siglo se registró una preocupación científica clínica, veterinaria y social creciente por la hidatidosis, comúnmente conocida como 'vejigas de agua'. La afección se provocaba por el contacto con verduras mal lavadas o animales infestados, especialmente perros en los que se alojaba la tenia equinococo en los intestinos que, a su vez, la adquirían por comer vísceras de ganado infes- 
${ }^{1}$ Las enfermedades infectocontagiosas consideradas como epidémicas eran difteria, sarampión, escarlatina, rubéola, varicela, gripe, poliomielitis, parotiditis, coqueluche, fiebre tifoidea, carbunclo, brucelosis, paludismo, alastrim. No se ha investigado aún la acción del gobierno para evitar las altas cifras de mortalidad que reportaba la gripe a lo largo del período. tadas. En el hombre, las vesículas se alojaban preferentemente en el pulmón y en el hígado generando invalidez e, incluso, la muerte.

Los reclamos de los doctos se dan a la saga de la conformación corporativa del área médica (Belmartino, Bloch, Persello, 1988). Hasta los años 30, la producción científica profundizaba en los aspectos morfológicos o sintomáticos cayendo en su 'espectacularización' por ser una enfermedad con efectos físicos deformantes. A fines de los años 30 y principios de los 40 , comienza una nueva visión que intenta relacionar la enfermedad con problemas sociales y económicos más estructurales (Ferro, 1961). Así, se refuerzan las demandas al Estado por parte de los médicos quienes entienden que estas preocupaciones deben ser solucionadas por medio de la aplicación de políticas sanitarias. La inestabilidad institucional del área de salud en la provincia de Buenos Aires durante el periodo entre 1943 y 1946 no logró encauzar esas acciones por lo que su proceso fue espasmódico y obtuvo pocos resultados.

En el ámbito nacional, en el Plan Analítico de Salud Pública (Pasp) de la Secretaría de Salud Pública (SSP) dirigida por Ramón Carrillo, se especificaban los lineamientos generales para la acción sanitaria. La SSP estaba enfocada en dotar a la Nación de una infra-estructura sanitaria a través de la edificación de hospitales, centros de higiene y dispensarios; ofrecer garantías legales para los problemas sociales y atacar enfermedades epidémicas y otras - que como la tuberculosis - tenían para el conjunto de las provincias mayor impacto. ${ }^{1} \mathrm{La}$ acción del Ministerio estuvo concentrada en: 1) codificar un sistema legal de acción específica contra las zoonosis; 2) coordinar reparticiones, dejando en manos de los ministerios provinciales las acciones concretas de erradicación y prevención. La contribución fundamental de la SSP fue dar apoyo a la educación sanitaria y a la acción propagandística acompañando los emprendimientos provinciales (La exposición..., jul. 1948; Valobra, Ramacciotti, 2004). En ese plan, salvo alguna zoonosis como la brucelosis, estas dolencias no fueron centrales en la propuesta nacional.

Durante la gobernación Mercante, el Ministerio de Salud Pública y Acción Social (MSPAS) bonaerense intentó mostrar su singularidad desplegando una tarea específica que, de modo general, coincidía con algunas preocupaciones que interpretaban que la salud era parte de la justicia social. Entre las enfermedades zoonóticas bonaerenses más preocupantes se encontraban la brucelosis, la rabia y la hidatidosis. (El lunes... 18 jul. 1947; Bocalandro, Esquivel, nov. 1949). La hidatidosis fue en cierto modo 'redescubierta' por el ministro de Salud de Mercante, Carlos Alberto Bocalandro, un médico castrense que ocupó las funciones ministeriales en el lapso comprendido entre el 29 de julio de 1947 al 4 de junio de 1952 y otorgó solidez a la gestión mercantiana a través de la continuidad y notable transparencia que destacaron a 
esta dependencia de otras. Bocalandro recuperó las demandas de larga data sobre la enfermedad al tiempo que orientó la prevención, vacunación, desinfección y tratamiento de animales y personas portadoras. Tanto en el caso de la rabia como en el de la hidatidosis, puede referirse una preocupación común: su contagio venía de la relación con un animal doméstico y por excelencia 'amigo del hombre', el perro, por lo que era importante generar conciencia de los peligros implicados en el contacto con ellos. Las zoonosis se convertían en un problema crucial que marcaría la singularidad de la intervención mercantiana en ese campo. El MSPAS desplegó diversas estrategias para terminar con la más preocupante endemia zoonótica de la región.

\section{Legitimación de la importancia de la hidatidosis: entre la estadística y la sensibilidad}

Legitimidad estadística

El afán estadístico tenía una larga tradición como práctica racionalizadora del estado moderno y el peronismo logró canalizarlo en instituciones y artefactos estadísticos concretos como el Censo de 1947 o el Censo sanitario de 1954 (Berrotarán, 2002, 2004). Para el gobierno, la estadística era una práctica de racionalización de la intervención estatal y fundamental para estructurar prácticas sanitarias (Argentina, 1949). La estadística constituyó también un basamento legitimante para la determinación de la hidatidosis como foco mórbido. La información cuantitativa sobre la enfermedad era "la más copiosa que existe en el mundo para un solo país y ... constituye un argumento más para llamar la atención sobre la impostergable urgencia de proceder enérgicamente contra tan temible parasitosis" (p.80). En efecto, a partir de un seguimiento estadístico se pudo delinear la distribución de la dolencia, su geografía y los alcances sobre la población humana y animal. Según las Memorias del MSPAS, la provincia de Buenos Aires tenía el triste honor de ser la región con más infectados de hidatidosis en el mundo. El MSPAS retomó las investigaciones de Alfredo Ferro (19011970), un médico oriundo de la zona de mayor infestación provincial - Azul - que en 1939, a influjo de su vínculo profesional con el afamado hidatólogo de Rouen, Félix Devé, había fundado el Instituto de Hidatidología de Azul y en 1941, la Asociación Internacional de Hidatidología junto al destacado cirujano uruguayo Velarde Pérez Fontana (1897-1975). Ferro demostraba que “desde 1935 ... los casos de hidatidosis humana alcanzan a la impresionante cantidad de varios miles... la mortalidad no desciende de un 10 a un 12\%" (Argentina, 1948, p.22). La provincia había acumulado, en poco más de un decenio, el mayor número de enfermos: más del 67\% entre 1935 y 1947 (4.186 casos) y, según las épocas, podía au- 
mentar (Crivellari, Mendy, 1949). Si bien no faltaron voces menos alarmistas frente a los guarismos, el gobierno de Mercante utilizó estos datos para legitimar su preocupación. Los dirigentes del Ministerio creyeron estar cerca de un avance exponencial de la enfermedad y reforzaron visiones apocalípticas del pasado que afirmaban que mientras la población se duplicaba, la enfermedad se cuadriplicaba (Argentina, 1948). No obstante, según las opiniones, no fue tanto el número de enfermos en sí lo que alarmaba sino el aserto de que la extensión de la enfermedad se registraba en el caso de la 'primera provincia argentina'. Ello afectó sensiblemente la visión de sí que tenían los gobernantes bonaerenses y fue un importante motor de acción.

Legitimidad socioeconómica y territorialidad rural

Como toda intervención, además de la lectura estadística, la práctica sanitaria estaba influida también por la concepción de la sociedad sobre la que se iba a intervenir. En este plano jugaron factores de orden simbólico, económico, social y político que conllevaron una particular construcción de la hidatidosis como preocupación de la política general: la revalorización de la producción agropecuaria y de la población rural.

Mercante afianzaba el sector rural teniendo en cuenta que en la provincia "la prioridad de la actividad agroganadera era indiscutible frente al desarrollo industrial que se estaba promoviendo desde el gobierno nacional" (Blanco, 2001). Además, recuperó la retórica de 'la tierra para el que la trabaja' y consideró necesario plasmar un tipo de intervención política estatal que socavara los conflictos de la clase rural (Inició..., jul. 1948). Los cambios institucionales durante su gestión dan cuenta de la jerarquización de las actividades agroganaderas por lo que las enfermedades en animales reportan interés al afectar la producción (Blanco, 2001).

En materia económica, la enfermedad arrojaba pérdidas importantes para el sector agropecuario afectando al $12 \%$ de la hacienda (Argentina, 1948, p.20). Ello atentaba contra el erario público y la economía privada por el decomiso de vísceras y animales infestados y por la esfumación de jornales de trabajo no cumplidos pero pagados a enfermos, material de curaciones, etc. (Argentina, 1948).

Entonces, por sus características, la hidatidosis se visualizó estrechamente asociada a los modos de explotación del capital pues, para su prevención y profilaxis, no sólo había que generar una conciencia sanitaria, sino también una infraestructura material que garantizara ciertas condiciones de higiene en los establecimientos destinados al faenamiento del ganado $y$, finalmente, implicaba una fuerte presencia del Estado para erradicarla mediante una intervención concienzuda. 
Con todo, lo económico no fue la única causa legitimante para la lucha antihidática. También se otorgó importancia a la situación social de los trabajadores rurales y sus condiciones de vida. Se inscribía así en la propuesta iniciada por Perón en el año 1943, durante su gestión en la cartera de Trabajo y Previsión, que cuajó en el Estatuto del Peón.

Desde el MSPAS se dirigió una nueva mirada al mapa sanitario apelando a términos geográficos y económicos. En primer lugar, destacaron que la enfermedad se concentraba en áreas relativamente muy ricas en términos productivos: la línea del paralelo de $35^{\circ}-$ en la que sólo se exceptuaban los países de clima tórrido. Asimismo, atañía al capital humano pues "una nación es fuerte en tanto los individuos que la componen son sanos y robustos" (Bocalandro, 1947-1948, p.18).

La jerarquización del sector rural fue acompañada de la aspiración de aumentar el nivel de vida de su población: “No puede ser... que los especialistas de enfermedades epidémicas sólo se hallen en las grandes ciudades" (No puede ser, 12 abr. 1948).

La justicia social sería llevada por medio de la intervención del estado a la población rural olvidada. La intervención sanitaria - así como la educativa - estaría llamada a jugar un importante papel.

Las enfermedades infectocontagiosas y preponderantemente urbanas habían sido hasta entonces objeto de mayor atención debido a que en esos ámbitos se concentraba la población de la provincia. Ello amplificaba la difusión de enfermedades infectocontagiosas adquiriendo rasgos epidémicos que cobraban la vida de miles de personas en breves lapsos de tiempo (Argentina, jul. 1949). En líneas generales, la revalorización de lo rural no fue la base de las propuestas de la intervención estatal. El caso de la denodada lucha de Salvador Mazza y Carlos Chagas por establecer la prioridad de la enfermedad transmitida por la vinchuca da cuenta de las posturas hegemónicas contra las que debían confrontar (Ramacciotti, otoño 2005; Delaporte, nov.1994-feb.1995). La asociación de lo rural con lo tradicional, no moderno, lo 'no blanco-aborigen-mestizo-pobre' y la intervención sanitaria era condicionante del interés. Incluso cuando la intervención sanitaria existía, iba acompañada de una sanción social y moral que llegaba a culpabilizar al enfermo (Scarzanella, 1999, jul.-dic. 2003).

Sin embargo, desde el MSPAS se quebró la lógica con la que se había organizado hasta entonces la intervención estatal. Se afirmó que los núcleos urbanos eran en superficie menos extensos que los rurales y que en estos la población no era despreciable: 1.000.000 de personas (Argentina, 1948). Además, se apelaba a una sensibilización frente a los problemas rurales que, en un discurso peronista afecto a las resoluciones dicotómicas en la oposición pasado-presente, no excluyó otras tales como lo rural-urbano. 
2 En este entramado, la salud encontró en la escuela un arma de educación sanitaria fundamental en los que el vitalismo campestre se unía a la noción de salud corporal y espiritual. Varios de estos aspectos se presentan en la Constitución de 1949, en su capítulo IV y en el Plan de Ruralización de la Enseñanza Primaria de los Niños Campesinos.
Si bien las políticas de Mercante no rompieron la visión dual urbano-rural, imprimieron una mirada distinta al tema al volver a localizar las intervenciones estatales en lo rural y señalar las interconexiones existentes entre lo cultural, social y económico. El planteo invertía la lógica sanitaria de las prácticas estatales hasta ese momento preocupadas por las consecuencias de las migraciones del campo a la ciudad y el impacto urbano. La propuesta mercantiana intentó satisfacer las necesidades sanitarias de los sectores rurales - visualizadas como un aspecto fundamental de su emigración - a fin de que no migraran. La nueva lógica territorial recuperaba - no sin torsiones - 'lo rural' como lugar identitario original de 'lo bonaerense' $y$, en otras oportunidades, de 'la argentinidad'. La población rural pasó a convertirse en un sujeto colectivo que, por sostener el potencial económico nacional, demandaba la intervención estatal. Esta idea se expresó tanto en la acción sanitaria como educativa (Maldones, mar. 1949; Mignone, 1949). ${ }^{2}$

Este repaso nos ha permitido comprender cuáles fueron los fundamentos de orden económico, médico y social que movieron al gobierno de la provincia a encarar la lucha contra la hidatidosis. Esto legitimaba, sin duda, una acción inmediata en la que el Estado tomara cartas en el asunto. Si bien había zonas de difícil resolución, el gobierno de Mercante asumió ese compromiso creando un aparato legal y una intervención estatal específica.

\section{Estrategias sanitarias mercantianas de lucha contra la hidatidosis}

A continuación diferenciaremos las estrategias de acción en la propuesta racionalizadora de la práctica sanitaria estatal en la lucha contra la hidatidosis que propició el gobierno mercantiano.

Las leyes y las instituciones como sustrato para una práctica racional

Los instrumentos legales normativos fueron los antecedentes inmediatos a la creación de instituciones antihidatídicas: "La lucha contra la zoonosis requiere y exige una acción tenaz y decisiva ..., con las nuevas armas de la ciencia contemporánea y con legislaciones novísimas, previsoras, prudentes..." (Argentina, 1948, p.16). La normativa fue un marco necesario donde inscribir las tareas sanitarias y diseñar instituciones específicas contra la hidatidosis.

La ley provincial 5116 de creación del MSPAS ya contemplaba la labor profiláctica para prevenir las zoonosis. Dependiendo de la Dirección General de Acción Médico Social, el artículo 27 de la ley 5116 creaba la División II. Como área ministerial, la División era la encargada de la lucha contra las enfermedades sociales entre las que se encontraba la hidatidosis junto al cáncer, la lepra, la 
3 La acción en este tema se basaba en la educación popular, restricciones a la circulación de perros y vacunación antirrábica preventiva de los perros. El segundo punto, que implicaba la "recolección de perros callejeros", generaba notables problemas pues "las reacciones populares contra los recolectores de perros" eran un "atentado contra las autoridades sanitarias" (Bocalandro, Esquivel, nov. 1949, p.549). brucelosis, las alergias y las venéreas (Maciel Crespo, Doménech, 1947-1948). La hidatidosis alcanzó notable relevancia como problema de estado pues constituía el tercer problema más importante de los cinco que se percibían como los que más gravemente aquejaban a la provincia. Los otros eran enfermedades de origen hídrico con carácter endémico, la alineación mental, la salud materno-infantil y la insuficiencia de camas para internación (Bocalandro, Annusi, 1947-1948).

La creciente importancia de la intervención antihidatídica tuvo consecuencias visibles en el recorrido institucional. En poco tiempo, el dispensario antihidatídico de Azul - la zona más afectada por la endemia - creado el 26 de agosto de 1947, fue transformado en División en 1949.

Retomando la ley 12.732 de 1941, a cuyo amparo se habían originado algunas iniciativas en el período anterior, se logró dotar a la hidatidosis de estatus de enfermedad de declaración obligatoria. En su ayuda fue sancionada la ley 5220 (octubre de 1947) que declaraba obligatoria la vacunación antirrábica. La normativa de profilaxis canina de la rabia incluyó un cronograma de vacunación que obligaba a los dueños del animal a vacunarlo cada seis meses $y$, además, incluía un tratamiento antihelmíntico que apuntaba a la profilaxis de la hidatidosis (artículo 3). Esta acción fue profundamente resistida por la población que rechazaba la vacunación y el tratamiento sobre todo porque las disposiciones consideraban el sacrificio del animal tanto al detectarse la rabia como cuando hubiera estado en contacto con otro rabioso (Revista..., 1948). Esto obligó al gobierno a reforzar las tareas de concientización y la captura de animales sin collar o bozal, lo cual generaba nuevas reacciones (Bocalandro, Esquivel, nov. 1949). ${ }^{3}$

En 1948 se creó la Dirección General de Ganadería y Medicina Veterinaria que entre sus objetivos planteaba la lucha contra la endemia. Asimismo, la creación del Instituto Biológico Tomás Perón garantizó al gobierno un abasto permanente de insumos para desparasitación de animales e investigación sobre medicamentos (Argentina, 1950).

Finalmente, numerosas labores del Plan Trienal de Trabajos Públicos, 1947-1949, consideraron como obras hidráulicas y mataderos rurales fundamentales para atender a esta zoonosis (Inició..., jul. 1948; Barba, Demaría Massey de Ferré, 1987).

Entonces, una profusa legislación iba acompañada de una complejización de las instancias institucionales que atenderían la endemia rural. Ello obedecía a un lógica racionalizadora en virtud de la cual el estado presuponía la formación de áreas político administrativas merced a las cuales se delimitaba un problema específico y se atendían las soluciones viables. El basamento legal sirvió como marco para estructurar acciones del estado. 
4 Durante el período que abordamos en este trabajo, 19461952, se realizó, además de los mencionados congresos, el IV Congreso de Santiago de Chile en 1952.

\section{Espacios de formación e intercambio}

El MSPAS propició la formación científica y la investigación sobre hidatidosis entre los miembros de su cuerpo técnico logrando, además, un importante apoyo del sector médico y las entidades agremiadas del área. Su intención se basaba en el convencimiento de que la formación de veterinarios, agrónomos y médicos permitiría la racionalización de la política sanitaria por medio de agentes técnicos de distintas especialidades con funciones delimitadas y complementarias (Argentina, 1948). En este sentido, el gobierno aspiraba a ser quien brindara unidad e interdisciplinariedad a la acción de cada disciplina, una verdadera "cruzada sanitaria integrada con elementos destacados de ambas ramas médicas para dilucidar en forma racional y completa la etiología, la patogenia y la profilaxis de muchas afecciones que, en varias especies, en determinadas zonas y circunstancias de causalidad influyen en la aparición de focos sépticos..." (p.16).

Asimismo, desde el MSPAS se apeló al sanitarismo supranacional trabajando estrechamente con médicos de otros países cuyo conocimiento en el tema podía iluminar su tarea. Para ello se apoyó en la experiencia de países hermanos como Brasil y Uruguay y favoreció congresos de la especialidad. El 1 de diciembre de 1948 se realizó el Primer Congreso de Zoonosis en la provincia de Buenos Aires. El Acto inaugural alcanzó envergadura oficial realizándose en la Cámara de Senadores con la presencia de delegados extranjeros, civiles, militares, legisladores nacionales y provinciales. Lo inauguró el gobernador de la provincia, Mercante, para "destacar la importancia de sus fines y beneficios para la salud pública" (Argentina, 1949, p.15).

El II Congreso de la Hidatidosis, realizado en Azul en 1948, continuó la tarea iniciada por el I Congreso de este tipo que en 1947 había reunido en Uruguay a la comunidad científica y técnico administrativa estatal. ${ }^{4}$ Asistieron especialistas latinoamericanos y europeos. La enfermedad amenazaba con convertirse en un problema internacional por su propagación en fronteras con Uruguay y Argentina, en la cual resultaba especialmente damnificado Brasil (Meneghetti, 1949). El contagio no respetaba fronteras y los acuerdos entre naciones se convirtieron en un instrumento para atender a ese esparcimiento. Así, para evitar el traspaso transcordillerano, Argentina y Chile firmaron un Acuerdo Sanitario en noviembre de 1948.

El Congreso de Azul marcó la centralidad que alcanzaba la hidatidosis para la agenda provincial, la voluntad férrea del gobierno de erradicarla y la intención de ampliar los aportes científicos (Dan a conocer..., 1948; Segundo Congreso..., 10 abr. 1948; Sociedad..., 8 abr. 1948). Fue para la provincia de Buenos Aires el 
puntapié inicial para lanzar un programa de acción antihidático de gran envergadura.

A su vez, el Congreso resultó de una gran variedad de propuestas para pensar la enfermedad y cómo atenderla.

El presidente de la comisión consultiva honoraria del Congreso y fundador de la Sociedad de Cirugía, José M. Jorge, hizo hincapié en que la "promiscuidad del hombre con el perro [era] factor fundamental de contaminación de la enfermedad" (Realizóse..., ene. 1948, p.67). Cuestionaba, de este modo, sobre las prácticas habituales en la población como foco de propagación de la enfermedad y demandaba una educación sanitaria que concientizara a las personas en la necesidad de erradicarla. Sin embargo, cabe destacar que esta condena a la relación de las personas con sus animales no fue unívoca. Médicos patagónicos rescataron que "el cariño del hombre de campo por su perro emana directamente del hecho de que le es imprescindible para cuidar de sus lanares" (p.72). Este punto de partida incidía en el tipo de campañas antihidáticas. En efecto, las realizadas en Buenos Aires se basaban en la desparasitación y educación sanitaria para evitar la promiscuidad con el animal y podía llegar a la captura del canino y su alejamiento del medio familiar. Sin embargo, las de la Patagonia focalizaban en la labor educativa respecto del cuidado de la higiene al entrar en relación con los animales, sin atacar la relación que consideraban base de la organización económica que giraba en el ganado lanar (p.72)). En este sentido, un problema 'técnico' acerca del modo de encarar los condicionantes socioculturales que se jugaban en la conformación del entramado sanitario estatal.

Asimismo, diferencias surgieron cuando se examinaron las técnicas quirúrgicas. Utilizadas para extirpar quistes de distintas partes del cuerpo humano infectadas, eran ampliamente desarrolladas por los argentinos, aunque criticadas por los uruguayos por ser muy invasoras por lo que se impulsaban métodos biológicos de tratamiento para reabsorción de las vejigas de agua (Realizóse..., ene. 1948, p.70).

La existencia de distintos métodos de detección de la enfermedad también generaba disputas las cuales se intentaron resolver con la asunción del método biológico de Appathie de reconocimiento de la hidatidosis con el fin de alcanzar estandarización (Rosas Costa, 1950).

También se observaron avances en el reconocimiento e interpretación radiológica de la enfermedad así como en los casos de quistes gigantes de pulmón.

Finalmente, no faltaron observaciones de tipo corporativas relacionadas con el reconocimiento a la labor de los veterinarios en los mataderos y los escalafones y la necesidad de que se ampliara el personal veterinario en las plantas pues el número de especialistas era insufi- 
ciente para la labor que se demandaba y la responsabilidad que tenían: certificar la salud de la población (Asociación..., mayo-jun. 1946).

En síntesis, en la búsqueda de la racionalidad sanitaria que combatiera la enfermedad, desde el MSPAS se diseñó una acción interministerial e interdisciplinaria amparada en la idea de que la equinococosis era una enfermedad zoonótica y demandaba la acción conjunta de médicos y veterinarios locales e internacionales y que el conocimiento científico era la mejor manera de afrontarla. Las ideas encontraron, no obstante, límites efectivos dados por los intereses que se jugaban en la erradicación de la enfermedad.

Las prácticas estatales en la erradicación y prevención de la enfermedad

Para la acción contra la hidatidosis era clave conocer, en una primera etapa, cuál era el alcance de la enfermedad y a quiénes afectaba. Para obtener una imagen lo más ajustada posible de la situación, el gobierno continuó con el censo hidático que desde 1934 se realizaba en la provincia y trató de ajustar algunas de las variables de información solicitadas allí. En un segundo momento, la sistematicidad de los tratamientos garantizaría que no se reinfectaran ni personas ni animales. Sin lugar a dudas, y a diferencia de lo sucedido hasta entonces, la acción antihidatídica durante el gobierno de Mercante estuvo centrada en la profilaxis canina a través de la desparasitación. En este sentido, el gobierno siguió las indicaciones del médico veterinario de origen alemán, dr. Francisco Rosenbusch, a la sazón fundador del Instituto Rosenbusch de Biología Experimental Agropecuaria S.A., que recomendaba en el tratamiento de los perros "un elemento cuya eficacia es completa: el bromhidrato de arecolina" (Realizóse..., ene. 1948, p.71).

Este era un camino arduo para el gobierno pues exigía una acción sistemática de tratamiento de los animales. Se proyectó un plan profiláctico con la edificación de dispensarios antihidatídicos tanto para tratamiento humano como canino. La acción en la zona rural se caracterizaba por la dispersión de la población por lo que el MSPAS desdobló sus acciones con esfuerzos exponenciales estableciendo laboratorios rodantes y vagones sanitarios para realizar las campañas (Con fines..., 1946). Se llegó a contar con 14 equipos móviles en las zonas de afectación. La tarea de los equipos móviles y dispensarios abarcaba el tratamiento de animales huéspedes de la teniasis (ganado y caninos). Asimismo, los perros eran vacunados contra la rabia y se entregaban las patentes de circulación si estaban en condiciones.

También se visitaban hogares para detectar personas que padecieran la enfermedad y para fortalecer la conciencia sanitaria (Argentina, 1950). Durante las campañas de profilaxis, las briga- 
das móviles contaban con empleados supervisados por un médico veterinario y, en algunos casos, jefe del dispensario local. Según informó el jefe de la campaña en Azul, Alfredo Ferro, en 1948 se habían recorrido 946.600 hectáreas, tratado a 1.583 perros, visitado 1.513 domicilios y examinado a 6.483 personas (Realizóse..., ene. 1948, p.71).

En los centros urbanos se efectuó un estudio de los mataderos con el fin de clasificarlos desde el punto de vista hidático e higiénico, en buenos, regulares y malos. Asimismo, se fiscalizó la labor de la inspección veterinaria proveyéndose a las municipalidades de una planilla mensual para anotar los decomisos por hidatidosis. Paulatinamente se fue ampliando la planta de dispensarios ubicados estratégicamente en las zonas más afectadas - Tapalqué, Miramar, Mar del Plata, Lobería y Patagones (Argentina, 1949, p.80). Cuando no era posible la construcción de un dispensario antihidatídico, se ubicaron salas dentro de los dispensarios polivalentes existentes.

Dado el doble carácter de la enfermedad, atacando personas y perros, los dispensarios se combinaron con perreras, baños de acaroína, cloacas y básculas para pesar animales. Se organizó un censo de perros de modo de conocer el estado, el lugar habitacional, las condiciones de circulación, etc. Veinticuatro comisiones de técnicos se encargaban de administrar el tratamiento antihelmíntico con el vermífugo conocido como bromhidrato de arecolina (Argentina, 1948). El tratamiento antiparasitario debía cumplir ciertos requisitos: obligatoriedad, universalidad, gratuidad, metódico, oficial, comodidad y asociación a otras profilaxis (por ejemplo, dedetización, desratización, antituberculosa y antibrucelósica). En este tratamiento, Ferro reconocía que era fundamental que los dueños de los animales tuvieran confianza en quienes lo ejecutaban y que ello debía propiciarse por todos los medios pues muchas veces, tal como sucedía con el tratamiento de la rabia, los dueños de perros se resistían a este tipo de intervención haciendo fracasar todo emprendimiento por falta de conciencia sanitaria (Ferro, 1948).

Se implementaron campañas de tratamiento antihelmíntico y se aprovechaba la ocasión en la que se reunían los habitantes para implementar breves actos educativos. En ella se apelaba a eliminar la carneada domiciliaria y evitar el suministro de vísceras infestadas a los perros. Como parte de las tareas de divulgación sanitaria, también se proyectaba una película realizada por el jefe de la lucha, el dr. Alfredo Ferro. Se organizaron campañas en escuelas en colaboración con la Dirección General de Escuelas, publicidad periodística, radial, mural y cinematográfica mediante el aparato proyector que poseía la división (Argentina, 1948, p.66), pues el cine se consideraba una herramienta didáctica importante. También era completada por clases en escuelas y cuarteles, afiches, letreros, carteles y relatos de carácter infantil (Mendy, 1950). 


\section{Limitaciones y alcances en la acción antihidática}

La gestión de Mercante se caracterizó por una férrea voluntad del estado orientada a dos aspectos: la recuperación del sujeto rural y la necesidad de modernizar el estado a través de la racionalización de las actividades. La compleja realidad que dibujaba el mapa de la hidatidosis fue por momentos inasible, pero el balance durante la dirección de Bocalandro resulta altamente positiva, sobre todo comparada con años posteriores.

En primer lugar, los alcances de la legislación rural promovida entre 1943 y 1946 y luego continuada por Mercante, tuvo limitaciones pues no atendía a una extensa mano de obra sin relación de dependencia que trabajaba estacionalmente en el campo. Siguieron existiendo personas que vivían en condiciones de pobreza extrema y que eran las principales víctimas de enfermedades como la hidatidosis. Según Graciela Agnese, el contraste de pobreza en la región más rica de la Argentina despertaba insistentes denuncias de los médicos rurales que comprendían que un buen número de los pobladores de esta zona

no tenían ningún tipo de cobertura de salud ni seguros de vida y, si bien en los hospitales se les brindaba atención médica gratuita, ante el fallecimiento, sus familias quedaban sometidas al más absoluto desamparo. Incluso no podían trasladar a las víctimas fatales a su lugar de origen y eran los gobiernos municipales los que, por pedido de los médicos, disponían el enterramiento en el cementerio local. (Agnese, 2007)

${ }^{5}$ En el sentido apuntado, el diario $E l$ Día denunciaba que "la falta de médicos, así como de profesionales de actividades afines, no es, como se ve, un mal exclusivo de zonas apartadas del interior del país. En la propia provincia de Buenos Aires y a no mucha distancia de su capital y del aglomerado metropolitano, hay núcleos de población tan pobremente atendidos que, en un momento dado, pueden quedar sin asistencia particular ni oficial..." (El servicio.... 16 jul. 1947).
Las limitaciones de la política sanitaria eran reconocidas por el mismo ministro Bocalandro (jun. 1949). ${ }^{5}$ En 1948, año de lanzamiento de la acción estatal contra la hidatidosis, un exceso de optimismo en la capacidad de racionalización de las tareas se convirtió en dificultad para las actividades y la evaluación de los logros. Las reflexiones en torno a la campaña de hidatidosis sostenían que "los pronósticos que se hicieron sobre la duración de la primera campaña fueron demasiado optimistas ya que recién para el mes de julio del corriente año se habrá cubierto por primera vez en su totalidad la zona de infestación máxima" (Argentina, 1949-1950, p.81). Para 1950, el MSPAS insistía: “Seguimos siendo uno de los centros de mayor infestación en el mundo" (Argentina, 1950, p.50, 51). Las excesivas expectativas impedían ponderar la importantísima disminución de casos de hidatidosis.

Un debate por demás extenso fue el que surgió cuando se propuso que la hidatidosis fuera considerada en las pólizas de accidentes de trabajo como enfermedad profesional o rural. Dado que la enfermedad hidatídica era un peligro público, el Estado debía proteger a quienes había atacado temporal o permanentemente (Jorge, 
Ferro, 1949; Serres, dic. 1949; Pérez Fontana, 1948). Gregorio Aráoz Alfaro, - eminente pediatra e higienista, ex presidente del Departamento Nacional de Higiene y de la sección eugenesia de la Sociedad Argentina de Biotipología, Eugenesia y Medicina Social - señaló que considerar la hidatidosis como enfermedad profesional

podría ser un medio más para convencer a los hacendados y patronos de que su propio interés se asocia al interés público, para decidirlas (sic) a prestar la mayor atención al grave asunto y a colaborar con perseverancia y decisión en las medidas profilácticas que han de conducir a detener y finalmente a extinguir esta enfermedad, cuya gran difusión es una ofensa a nuestra cultura (Jorge, Ferro, 1949, p.290).

Sin embargo, surgían inconvenientes acerca de cómo determinar qué patrón era responsable por la indemnización pues la aparición de la enfermedad no era fácilmente detectable y la fecha de diagnóstico no era la de su aparición. Para el médico, veterinario y farmacéutico Bernardo Houssay (1887-1971), los empleadores tenían una responsabilidad en cuanto a las condiciones sanitarias de labor y a la venta de animales enfermos y debía multárselos, pero no le era imputable la falta de hábitos sanitarios de sus empleados. El delegado de la Sociedad Rural, Pedro Hardoy, consideró injusto recargar a los ganaderos con imputaciones y relativizó la enfermedad como accidente de trabajo pues la extensión de la misma no amparaba tal supuesto. El destacado cirujano Marcelino Herreras Vegas (1875-1958) optó por la prudencia e impulsó la educación sanitaria para el decrecimiento de la enfermedad. Asimismo, las consideraciones acerca del modo de infestación atañían también a la familia de los trabajadores rurales y era difícil determinar allí cuál era el grado de responsabilidad del empleador, aún cuando fuera de suyo que la familia vivía con el peón rural en la zona en la que trabajaba. Contrariado ante esas respuestas, el dr. Jorge, impulsor del proyecto, indicó que muchos de los médicos más ilustres de la Argentina habían señalado el rol comprometido que tenían los empleadores rurales en esta enfermedad y concluyó afirmando que las sugestiones que realizaba con el dr. Ferro eran "muy justas y serán muy útiles cuando llegue a establecerse la responsabilidad de los ganaderos y el gobierno tome el puesto que le corresponde" (Jorge, Ferro, dic. 1949, p.293).

Finalmente, la responsabilidad debía ser amplia pues los estudios demostraban que las familias de los peones estaban tan comprometidas en su salud como ellos (Jorge, Ferro, 1949). Sin embargo, un año después, Ferro se retractó de responsabilizar a los patrones rurales, ya que como afirmaban sus representantes de la Asociación Rural, estaban dispuestos a luchar contra una enfermedad que también los atacaba a ellos. Evidentemente, los poderes económicos 
constituidos en la provincia bonaerense ponían un coto a potenciales respuestas.

Estos límites o reveses no obstan para reconocer que la acción mercantiana fue exitosa en la lucha contra la hidatidosis. Ello se evidencia en la comparación de la gobernación de Mercante con la de su sucesor, Carlos Aloé (1952-1955). Los problemas entre Mercante y Perón llevaron al ocaso la estrella política del gobernador bonaerense. Aloé se posicionó en ese eje para construir su perfil en detrimento de aquel. Sin embargo, ni la comparación general ni la particular logran opacar la intervención mercantiana.

El gobierno de Mercante había dejado en claro que debía realizarse una acción sistemática y concienzuda contra la enfermedad de modo de no retroceder en los logros obtenidos. Durante el gobierno mercantiano, la labor fue sumamente intensa: en 1948 se habían tratado un total de 78.014 animales, mientras que en 1952, en el siguiente período de gobierno, sólo 41.132; y, en el caso de visitas domiciliarias durante la gobernación mercantiana se asistía a un número sensiblemente mayor (Argentina, 1949, 1952). Sin embargo, el gobierno de Aloé, sumido en un momento de crisis económica, recortó aún más las tareas de la División de Azul (Argentina, 1952). Las actividades de prevención en la gobernación de Aloé se retrajeron

dentro de los límites que los medios lo han permitido, habiendo suplido la acción tratativa antihélmica canina que no pudo llevarse a cabo, con otro procedimiento que si bien no llena las ventajas que la práctica de la misma acarrea, constituye un valioso complemento... Si se trata de la faz educacional para hacer llegar la noción del peligro que importa para la salud, no se ha escatimado esfuerzos y para lograr este objeto, nuestros limitados recursos se han volcado de lleno (Argentina, 1953, p.271; subrayado por mi).

La educación sanitaria era importantísima, pero no podía ser la base exclusiva de la rehabilitación de los animales infestados.

El año de 1952 marca los primeros logros visibles en la reducción de infestación humana y animal durante la gestión peronista, coronamiento a los esfuerzos realizados por erradicar la hidatidosis durante el gobierno de Domingo Mercante. Los gobiernos posteriores no lograrían ponerse a la altura de la labor desplegada entonces (Argentina, 1956).

\section{Consideraciones finales}

Este trabajo es un breve ejercicio que intenta pensar cómo las voluntades político ideológicas juegan detrás de la demarcación de ciertas fronteras sociales. En este caso, la de la salud-enfermedad. El gobierno de Mercante mostró una acción vigorosa y con exce- 
AGRADECIMIENTOS

Agradezco

especialmente a Karina

Ramacciotti y a Javier

Balsa la generosidad

con las fuentes; a

Eugenia Bordagaray,

la inestimable

colaboración en el

trabajo de archivo; a

Nadia Ledesma

Prietto, la esmerada

edición y a Karina, nuevamente, el aporte de ideas y

producciones para la realización de este trabajo.

\section{BIBLIOGRAFÍA}

Agnese, Graciela 2007

Argentina

Argentina

Argentina

Argentina mayo 1949

Argentina 1949

Argentina 1948 lentes resultados en la erradicación de la hidatidosis. Problemas de índole económica no fueron suficientes para justificar la intervención estatal y las consideraciones sociales y humanitarias surgieron como sustento locuaz de la práctica estatal. El gobierno peronista que sobrevino luego de las elecciones de 1951 no continuó la labor desarrollada en el área invocando distintos motivos para justificar su inacción. Algo similar acaeció en los gobiernos posteriores al golpe de 1955.

La historia regional y/o provincial ha intentado aportar matices a una visión bastante unívoca de los procesos históricos. Ello es particularmente cierto en relación a las interpretaciones acerca de la acción gubernativa durante el peronismo que ha sido caracterizada monolíticamente.

La regionalización de las políticas sanitarias y la salud rural aún espera aportes que superen el estadio inicial de las investigaciones. El análisis de la hidatidosis en la provincia de Buenos Aires se presentó como una oportunidad para destacar la singularidad de la acción provincial en esta área al tiempo que se apreció cómo el Estado es una estructura en construcción atravesada por conflictos e intereses en pugna no sólo en la estructura estatal misma sino en sus interconexiones con la sociedad civil. Sin duda, y apuntando a nuevos desafíos, esta investigación adhiere a la idea de la existencia de una política mercantiana recortada y diferenciada de los primeros gobiernos peronistas tal como se puede deducir de la acción antihidatídica que estudiamos.

El doctor Rodolfo Arribálzaga, un médico rural entre investigadores y peones. In: Álvarez, Adriana; Carbonetti, Adrián (Comp.). Saberes y prácticas médicas en la Argentina: un recorrido por historias de vida. Mar del Plata: Grupo HISA (Universidad Nacional de Mar del Plata); CEA (Universidad Nacional de Córdoba). En prensa.

Ministerio de Salud Pública y Acción Social de la Provincia de Buenos Aires. Memorias. Buenos Aires: MSPASPB.

Ministerio de Salud Pública y Acción Social de la Provincia de Buenos Aires. Memorias. Buenos Aires: MSPASPB.

Ministerio de Salud Pública y Acción Social de la Provincia de Buenos Aires. Boletín Bases para una lucha epidemiológica, Buenos Aires.

Ministerio de Salud Pública y Acción Social de la Provincia de Buenos Aires. Boletín Sanidad y Estadística Vital, Buenos Aires.

Ministerio de Salud Pública y Acción Social de la Provincia de Buenos Aires. Memorias. Buenos Aires: MSPASPB.

Ministerio de Salud Pública y Acción Social de la Provincia de Buenos Aires. Memorias. Buenos Aires: MSPASPB. 


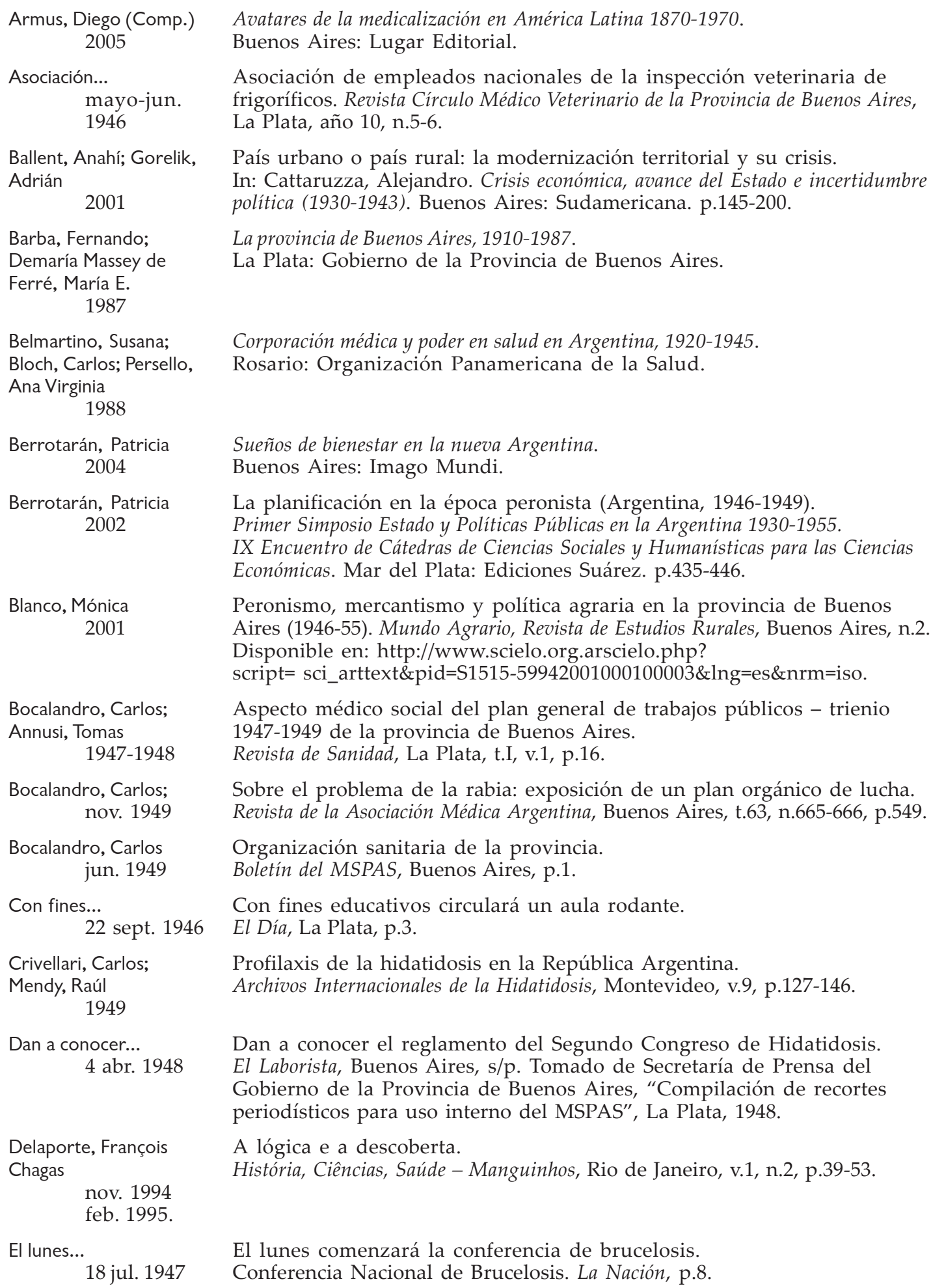


ADRIANA VALOBRA

El servicio...

16 jul. 1947

Ferro, Alfredo

1961

Ferro, Alfredo

1948

Gribaudi, Mauricio s.d.

Hizo declaraciones..

11 abr. 1948

Inició...

jul. 1948

Jorge, José; Ferro, Alfredo

dic. 1949

La exposición...

jul. 1948

La política...

jul. 1948

Maciel Crespo, F. A.;

Doménech, Armando 1947-1948

Maldones, Estanislao mar. 1949

Mendy, Raúl

1950

Mendy, Raúl Martín 1948

Meneghetti, Mario 1949

Mignone, Emilio 1949

No puede ser 12 abr. 1948

Pérez Fontana, Velarde 1948

Perspectivas... 1947-1948

Ramacciotti, Karina otoño 2005
El servicio médico en las pequeñas poblaciones.

El Día, La Plata, s/p. Tomado de Secretaría de Prensa del Gobierno de la Provincia de Buenos Aires, "Compilación de recortes periodísticos para uso interno del MSPAS", La Plata, 1947.

Problemas argentinos: la hidatidosis, problema sanitario y económico del quiste hidático a la zoonosis hidática. Revista de la Universidad, La Plata, n.13, p.121-140.

El tratamiento antiparasitario de los perros en la profilaxis de la hidatidosis rural. Archivos Internacionales de la Hidatidosis, Montevideo, v.8, p.133-137.

L'action publique dans les domaines sanitaire et social en France 1800a 1900. (Mimeo).

Hizo declaraciones el titular de la salud pública bonaerense.

La Época, Buenos Aires, p.211. Tomado de Secretaría de Prensa del Gobierno de la Provincia de Buenos Aires, "Compilación de recortes periodísticos para uso interno del MSPAS", La Plata, 1948.

Inició el gobierno bonaerense las nuevas normas impositivas.

Revista La Chacra, La Plata, p.185.

Hidatidosis. Enfermedad invalidizante.

Debe figurar entre las enfermedades profesionales $\mathrm{u}$ ocupacionales. Archivos Internacionales de la Hidatidosis, Montevideo, v.9, p.283-293.

La exposición de higiene en ASSP.

Archivos de la Secretaría de Salud Pública, v. 4, n.1, p.XXXIX.

La política agraria en la provincia de Buenos Aires.

Revista La Chacra, La Plata, p.182.

Estado actual, enfoque y perspectivas de la asistencia médico social en la provincia de Buenos Aires. Revista de Sanidad, La Plata, t.1, v.1, p.48.

Homenaje a la nueva constitución.

Revista de Educación, Buenos Aires, p.89.

Planificación de las campañas sanitarias.

Archivos Internacionales de la Hidatidosis, Paraná, v. 10, p.177-182.

Planificación de las campañas sanitarias en el Ministerio de Salud de Entre Ríos. Archivos Internacionales de la Hidatidosis, Montevideo, v.10, p.178.

Importancia de los convenios internacionales en la lucha contra la hidatidosis. Archivos Internacionales de Hidatidosis, Montevideo, v.9, p.35-37.

La educación en la constitución de la provincia de Buenos Aires.

Revista de Educación, Buenos Aires, n.5, p.25.

El Líder, v. 52, p.1.

Consideraciones sobre el seguro de hidatidosis.

Archivos Internacionales de la Hidatidosis, Montevideo, v.8, p.134-436.

Perspectivas de la asistencia médico social en la provincia de Buenos Aires. Revista de Sanidad, La Plata, , t.1, v.1, p.48.

Biografía de Salvador Mazza.

Médicos y Medicinas en la Historia, Buenos Aires, n.14, p.26-27. 
Realizóse... ene. 1948

Revista... 1948

Rosas Costa, Gustavo 1950

Scarzanella, Eugenia jul.-dic. 2003

Scarzanella, Eugenia 1999

Segundo Congreso... 10 abr. 1948

Serres, José

dic. 1949

Sociedad... 8 abr. 1948

Valobra, Adriana 2005

Valobra, Adriana; Ramacciotti, Karina 2004
Realizóse en Azul el Segundo Congreso Internacional de Hidatidosis. Archivos de la Secretaría de Salud Pública de la Nación, Buenos Aires, v.2, n.14.

Revista del Círculo Médico Veterinario de la Provincia de Buenos Aires. Buenos Aires, año 12, n.1-12.

Aspectos de la reacción inmunitaria en la hidatidosis y conveniencia de estandarizar sus métodos de diagnóstico biológico. Archivo Internacional de Hidatidología, Montevideo, v.10, p.197-200.

Los pibes en el Palacio de Ginebra: las investigaciones de la Sociedad de las Naciones sobre la infancia latinoamericana (1925-1939). Estudios Interdisciplinarios de América Latina y el Caribe, Tel Aviv, v.14, n.2. Disponible en: http://www.tau.ac.il/eial/XIV_2/scarzane.html.

Ni gringos ni indios: inmigración, criminalidad y racismo en Argentina, 1890-1940. Bernal: Universidad Nacional de Quilmes.

Segundo Congreso de Hidatidosis. El Plata, La Plata, s/p. Tomado de Secretaría de Prensa del Gobierno de la Provincia de Buenos Aires, "Compilación de recortes periodísticos para uso interno del MSPAS", La Plata, 1948.

La hidatidosis equinococósica y la ley no 9688 de responsabilidad por accidentes del trabajo. Archivos Internacionales de la Hidatidosis, Montevideo, v.9, p.437-439.

Sociedad Internacional de Hidatidología. Opinión, La Plata, p.83.

Tomado de Secretaría de Prensa del Gobierno de la Provincia de Buenos Aires, "Compilación de recortes periodísticos para uso interno del MSPAS", La Plata, 1948.

De cronopios y de famas: la atención del binomio madre-hijo en la política sanitaria bonaerense durante la gobernación de Domingo Alfredo Mercante, 1946 y 1952. In: Panella, Claudio (Comp.). El gobierno de Domingo Mercante: un caso de peronismo provincial. La Plata: Archivo Histórico de la Provincia de Buenos Aires. p.129-172.

'Plasmar la raza fuerte...': relaciones de género en la propaganda sanitaria de la Secretaría de Salud Pública de la Argentina, 1947-1949. In: Valobra, Adriana; Ramacciotti, Karina (Comp.). Generando el peronismo: estudios de cultura, política y género.

Buenos Aires: Proyecto Editorial. 